\title{
Growing Evidence for the Impact of Air Pollution on Depression
}

\author{
Naureen A. Ali, BSc ${ }^{1}$ Adeel Khoja, MBBS, MSc $^{2}$ \\ ${ }^{1}$ School of Nursing and Midwifery, Aga Khan University, Karachi, Pakistan ${ }^{2}$ Department of Medicine, Aga Khan University, Karachi, Pakistan
}

\section{TO THE EDITOR}

The World Health Organization (WHO) estimates that 9 of every 10 people worldwide inhale polluted air, and exposure to polluted air is accountable for 7 million deaths annually. ${ }^{1}$ Studies have associated the adverse effects of air pollution with respiratory, cardiovascular, and neurovascular diseases. ${ }^{2}$ Air pollutants can also cause serious neurocognitive effects - ranging from behavioral variations to neurodegenerative disorders - that ultimately can have devastating effects on mental health. ${ }^{3,4}$ WHO has taken the initiative to include mental health in the domain of noncommunicable diseases (NCDs), as mental health is a strong risk factor for NCDs. ${ }^{5}$

Mental disorders such as depression affect more than 300 million people worldwide; a rise of $>18 \%$ was reported between 2005 and $2015 .{ }^{5}$ Data from the World Health Survey across 60 countries show that $9.3 \%-23 \%$ of people have a chronic physical disease and depression. ${ }^{6}$ Globally and regionally, depression is the tenth leading cause of disabilityadjusted life years (DALYs). A DALY equates to 1 lost year of healthy life. By 2030, depression is expected to be among the 3 principal causes of DALYs. ${ }^{7}$

According to data from China published in 2018, every 1 standard deviation rise in particulate matter over an average PM2.5 concentration increases the likelihood of having mental illness (including depression) by $6.67 \%$, translating to an annual medical expense of $\$ 22.88$ billion USD. ${ }^{4}$ MohanKumar et al found that air pollutants, specifically particulate matter, induce inflammation and oxidative stress in the brain that can lead to the manifestation of depression. ${ }^{2}$ Vert et al found that the rate of depression was 2 times higher for each $10 \mu \mathrm{g} / \mathrm{m}^{3}$ increase in the nitric oxide level. ${ }^{8}$ Szyszkowicz et al reported a $7.2 \%$ increase in the risk of emergency department visits for depressive episodes with every $19.4 \mu \mathrm{g} / \mathrm{m}^{3}$ of PM10 concentration. ${ }^{3}$

These findings have important implications because most of the world's population resides in areas where particulate matter concentrations are greater than the WHO guidelines, ${ }^{1}$ and the association between air pollution and depression cannot be ignored. Public policies and individual actions are essential to reduce the effect of air pollution. Examples include creating green spaces and urban forestation for eliminating air toxins, avoiding exercising near crowded roads and streets, and improving household ventilation by substituting low-emission cook stoves for traditional solid household fuel.

Further research into the effect of air pollution on mental health is warranted through public-private partnerships.

\section{REFERENCES}

1. Air pollution key facts. World Health Organization (WHO). www.who.int/news-room/air-pollution. Accessed January 16, 2019.

2. MohanKumar SM, Campbell A, Block M, Veronesi B. Particulate matter, oxidative stress and neurotoxicity. Neurotoxicology. 2008 May;29(3):479-488. doi: 10.1016/j.neuro.2007.12.004.

3. Szyszkowicz M, Rowe BH, Colman I. Air pollution and daily emergency department visits for depression. Int J Occup Med Environ Health. 2009;22(4):355-362. doi: 10.2478/v10001-009-0031-6.

4. Chen S, Oliva P, Zhang P. Air pollution and mental health: evidence from China. The National Bureau of Economic Research. www.nber.org/papers/w24686. Published June 2018. Accessed February 5, 2019.

5. Other dimensions of the NCD crisis: from mental health, ageing, dementia and malnutrition to deaths on the roads, violence and disability. World Health Organization (WHO). www.who.int/ publications/10-year-review/ncd-other-dimensions/en/. Accessed January 16, 2019.

6. Moussavi S, Chatterji S, Verdes E, Tandon A, Patel V, Ustun B. Depression, chronic diseases, and decrements in health: results from the World Health Surveys. Lancet. 2007 Sep 8;370(9590):851-858.

7. Depression. World Health Organization (WHO). www.emro.who.int/health-topics/depression/index.html. Accessed January 16, 2019.

8. Vert C, Sánchez-Benavides G, Martínez D, et al. Effect of long-term exposure to air pollution on anxiety and depression in adults: a cross-sectional study. Int J Hyg Environ Health. 2017 Aug;220(6):1074-1080. doi: 10.1016/j.ijheh.2017.06.009. 